\title{
Chronic hepatitis B patients with high liver fibrosis levels should receive antiviral treatment
}

\author{
XU LI, QINGLONG JIN, HONGQIN XU, ZETIAN ZHANG, HONGJIE ZHOU, \\ DONGQING YAN, DONGMEI LI, PUJUN GAO and JUNQI NIU
}

\begin{abstract}
Department of Hepatology, The First Hospital of Jilin University, Changchun, Jilin 130021, P.R. China
\end{abstract}
Received February 6, 2016; Accepted February 10, 2017

DOI: $10.3892 /$ etm.2017.4422

\begin{abstract}
The present study aimed to evaluate improvements in liver stiffness (LS) measured by transient elastography (TE) and associated factors in Chinese patients with chronic hepatitis B (CHB) treated with entecavir (ETV). A total of 190 consecutive CHB patients who received entecavir therapy and two LS measurements (LSMs) were enrolled in this retrospective study (average age, 47 years; 137 males [72.1\%]), including 111 patients without liver cirrhosis (group 0) and 79 patients with liver cirrhosis (group 1). Each patient received LSM twice with an interval of six months. Normalized aspartate aminotransferase (AST) levels were accompanied by a significant reduction in LSM values $(\mathrm{P}<0.001)$ in each group. Multivariate analysis revealed that a higher initial LS value in groups 0 and 1, and a higher prothrombin time activity in group 1 was associated with a greater decline of the LS value. Higher initial quantitative hepatitis B surface antigen (qHBsAg) levels were correlated with a greater decline of the qHBsAg value in the two groups. In conclusion, LS values in CHB patients significantly improved after 24 weeks of entecavir therapy. The results suggested that higher LSM values at baseline contributed to a greater regression of LSM, and higher initial qHBsAg values could lead to greater qHBsAg reduction during ETV therapy. Extrapolating our results, we might be able to consider $\mathrm{CHB}$ patients who have higher initial LSM values or have higher levels of qHBsAg values when enrolled would have more benefit during ETV treatment.
\end{abstract}

\section{Introduction}

Hepatitis B virus (HBV) chronically infects 240 million people worldwide and remains a severe threat to public health. Chronic hepatitis $\mathrm{B}(\mathrm{CHB})$ can advance to cirrhosis

Correspondence to: Professor Pujun Gao or Professor Junqi Niu, Department of Hepatology, The First Hospital of Jilin University, 71 Xinmin Street, Changchun, Jilin 130021, P.R. China

E-mail: gpj0411@163.com

E-mail: 15143166188@139.com

Key words: liver fibrosis, qHBsAg, entecavir, chronic hepatitis B and hepatocellular carcinoma. CHB-associated complications account for $\sim 700,000$ mortalities per year. The ultimate goal for treating CHB is to block the advancement of chronic liver injury to the stages of cirrhosis, hepatic decompensation and/or hepatocellular carcinoma (HCC). Thus, an early and accurate diagnosis of fibrosis stages is important for assessing prognosis (1-3).

Liver biopsy provides tissue for assessing liver pathology, but its applications are limited due to its invasiveness, patient discomfort, sampling errors, inter-observer variation and risk for internal bleeding complications.

In recent years, there has been increasing interest in estimating liver fibrosis using transient elastography (TE), a non-invasive method. TE utilizes an ultrasound (US) transducer attached to the axis of the vibrator that generates pulsation featured with a mild amplitude and low frequency $(50 \mathrm{~Hz})$. This resultant elastic shear wave transmits through the liver. Detection based on US pulse-echo tracks the dissemination of the shear wave and computes its velocity, which is proportional to liver tissue stiffness. The stiffer the tissue, the quicker the shear wave is transmitted $(4,5)$. TE is a noninvasive, timesaving and reproducible method for assessing liver stiffness (LS) and it is well tolerated by most patients $(6,7)$.

Entecavir (ETV), a new-generation nucleoside analogue, profoundly suppresses HBV DNA replication and has been widely prescribed for treating $\mathrm{CHB}$ due to its high efficacy. Clinical evidence suggested that fibrosis, particularly in the early stage, may in fact be reversed by effective ETV therapy. However, the efficacy of ETV therapy in blocking the progression of liver injury and reversing fibrosis in CHB in Chinese populations has only been evaluated by few studies.

The present study evaluated the effects of ETV treatment on viral replication, liver injury and hepatic fibrosis in 190 Chinese CHB patients who received LS measurement (LSM) twice using fibroscan at baseline and week 24 of the treatment. LSM values in patients significantly improved after 24 weeks of ETV therapy.

\section{Patients and methods}

Patients. A total of 218 consecutive Chinese CHB patients, who had been hepatitis B surface antigen-positive for $\geq 6$ months, were enrolled in the present study from November 2013 to March 2015. The Child Pugh Score of all of the enrolled 
patients was $<9$ and the patients were aged 18-65 years. These patients received at least two LSMs while being administered oral ETV at the First Hospital of Jilin University (Changchun, China).

The exclusion criteria adopted were as follows: i) Co-infection with human immunodeficiency virus or hepatitis $\mathrm{C}$ virus (HCV); ii) excessive alcohol consumption or drug abuse; iii) alanine transaminase (ALT) $>100$ IU/l or aspartate transaminase (AST) >100 IU/1 at baseline; iv) hepatocellular carcinoma suggested upon the first abdominal ultrasound; v) history of ascites, upper gastrointestinal hemorrhage, hepatic encephalopathy, infection, electrolyte disturbances or Child Pugh Score $\geq 9$; vi) history of diabetes, hypertension or other metabolic conditions. Finally, 190 patients with CHB were enrolled.

Upon enrollment, each patient was subjected to an initial LSM and a second one followed $\sim 6$ months later. Liver cirrhosis was diagnosed based on US.

The recruitment of human subjects and the study protocol were approved by the Independent Institutional Review Board of the First Hospital of Jilin University (Changchun, China). Written informed consent was signed by each participant prior to enrollment.

Laboratory tests. All biochemical, serological and virological examinations were performed within five days prior to LSM. The ARCHITECT hepatitis B surface antigen (HBsAg) assay (Abbott, Chicago, IL, USA) was used to assess quantitative (q) HBsAg. Serum HBV DNA levels were examined by using the COBAS TaqMan HBV test with the High Pure System (Roche Molecular Systems, Inc., Pleasanton, CA, USA) at a lower detection limit of $15 \mathrm{IU} / \mathrm{ml}$. Serum HBV DNA levels were expressed as $\log 10$ units. qHBsAg and serum HBV DNA levels were established using blood samples collected upon admission.

LSM. Patients who had fasted overnight were subjected to LSM by transient elastography (TE) with an M-probe (FibroScan; Echosens, Paris, France). A minimum of 10 valid measurements were acquired from each patient. LSM values were considered valid and entered into the final analysis only if the success rate of LSM reached $>60 \%$. Calculated median values of the valid measurements (expressed in $\mathrm{kPa}$ ) reflected the relative LS for each patient at a specific time-point.

Statistical analysis. Quantitative differences in LSM value changes between two points were analyzed by Student's t-test. Percentages were weighed by Chi-square test. Continuous variables were expressed as the mean \pm standard deviation. Independent factors associated with LS were individually ascertained through stepwise multiple linear regression models. All tests were two-sided, and $\mathrm{P}<0.05$ was considered to indicate a statistically significant difference between values. SPSS v.23.0 (International Business Machines Corp., Armonk, NY, USA) was used for all statistical analyses.

\section{Results}

Patient characteristics at baseline. Clinical and laboratory characteristics of participants at baseline and follow-up are shown in Table I. A total of $190 \mathrm{CHB}$ patients treated with ETV were enrolled in the present study, including 137 male and 53 female patients, and the mean age was 47 years. The mean duration of antiviral treatment prior to initial LSM was 484 days. Patients were assigned to group 0 when liver cirrhosis was absent $(n=111)$ and to group 1 when liver cirrhosis was present $(n=79)$. No significant difference was detected in the demographic characteristics, including gender, $\mathrm{HBeAg}$-negative rate and family history of HBV infection between the two groups before baseline LSM. In addition, no significant difference was detected in the baseline levels of ALT, $\alpha$-fetoprotein (AFP), serum creatinine $(\mathrm{Cr})$, blood urea nitrogen $(\mathrm{BUN})$ and triglycerides between the two groups. Furthermore, the mean age in group 1 was higher than that in group 0 . At baseline, group 0 showed higher qHBsAg and HBV DNA titers, platelet count (PLT), albumin (ALB) and prothrombin time activity (PTA) than group 1, while initial AST and total bilirubin (TBIL) were higher in group 1. In addition, LSM values in group 1 were higher compared to those in group 0 . Furthermore, the therapy course in group 0 was longer than that in group 1.

Changes in liver biochemical markers and LS value. Biochemical markers significantly improved after patients in group 0 underwent ETV therapy (Table I). For instance, initial and follow-up levels were $32.83 \pm 14.30$ and $24.20 \pm 9.27$ IU/1 for AST $(\mathrm{P}<0.001), 18.09 \pm 60.50$ and $3.26 \pm 1.94 \mathrm{ng} / \mathrm{ml}$ for AFP $(\mathrm{P}=0.010), 89.60 \pm 11.12$ and $93.49 \pm 12.60 \%$ for $\mathrm{PTA}(\mathrm{P}=0.001)$, $26.13 \pm 19.89$ and $26.69 \pm 15.82 \mathrm{IU} / 1$ for ALT $(\mathrm{P}=0.763)$, $20.10 \pm 11.55$ and $18.29 \pm 7.87 \mu \mathrm{mol} / 1$ for TBIL $(\mathrm{P}=0.063)$, $2.07 \pm 1.59$ and $1.12 \pm 1.51 \log \mathrm{IU} / 1$ for HBV DNA $(\mathrm{P}<0.001)$, and 2,991.54 \pm 5247.24 and 2,597.24 $\pm 5846.97 \mathrm{IU} / \mathrm{ml}$ for $\mathrm{qHBsAg}$ $(\mathrm{P}=0.488)$, respectively. The mean $\mathrm{LS}$ value at baseline was $14.84 \pm 9.42 \mathrm{kPa}$, which decreased to $8.36 \pm 4.88 \mathrm{kPa}$.

Initial and follow-up levels in group 1 were $39.91 \pm 15.08$ and $32.34 \pm 11.04 \mathrm{IU} / 1$ for $\mathrm{AST}(\mathrm{P}<0.001), 31.91 \pm 17.01$ and $33.97 \pm 17.45 \mathrm{IU} / 1$ for ALT $(\mathrm{P}=0.257), 24.39 \pm 12.63$ and $24.91 \pm 11.26 \mu \mathrm{mol} / 1$ for TBIL $(\mathrm{P}=0.675), 76.39 \pm 15.03$ and $80.90 \pm 16.42 \%$ for PTA $(\mathrm{P}<0.001), 11.75 \pm 29.64$ and $11.72 \pm 68.30 \mathrm{ng} / \mathrm{ml}$ for $\mathrm{AFP}(\mathrm{P}=0.998), 2.20 \pm 1.38$ and $0.88 \pm 1.19$ $\log \mathrm{IU} / 1$ for HBV DNA $(\mathrm{P}<0.001)$ and $1,240.17 \pm 1387.61$ and $1,066.22 \pm 1184.79 \mathrm{IU} / \mathrm{ml}$ for $\mathrm{qHBsAg}(\mathrm{P}=0.067)$, respectively. LS values significantly decreased from $21.08 \pm 8.55$ to $16.01 \pm 10.60 \mathrm{kPa}(\mathrm{P}<0.001)$ during the 24 weeks of ETV therapy.

Parameters correlated with the improvement of LS. Univariate analysis suggested that factors indicating a greater decline of the LS value in group 0 included higher initial LS values $(\mathrm{P}<0.001)$, higher initial AFP values $(\mathrm{P}=0.001)$, lower initial serum ALB $(\mathrm{P}=0.029)$, lower initial BUN $(\mathrm{P}=0.031)$ and shorter duration of antiviral therapy prior to initial LSM $(\mathrm{P}=0.006)$. Furthermore, there was a positive correlation between the changes of LS values and the changes of HBV DNA after 24 weeks of ETV therapy $(\mathrm{P}=0.005)$. The abovementioned factors were considered for entry in multivariate analysis. After adjusting for potential confounders, higher initial LS values were independently associated with a significant reduction in LS values ( $\mathrm{P}<0.001$; Table II). 
Table I. Clinical and laboratory findings in the two groups.

\begin{tabular}{|c|c|c|c|}
\hline Characteristic & Group $0(n=111)$ & Group $1(\mathrm{n}=79)$ & P-value \\
\hline Age, years & $45.94 \pm 9.76$ & $49.61 \pm 9.66$ & 0.011 \\
\hline Male gender, n (\%) & $79(71)$ & $58(73)$ & 0.734 \\
\hline HBeAg (positive/negative) & $54 / 57$ & $32 / 47$ & 0.266 \\
\hline Family HBV infection history (yes/no) & $56 / 55$ & $36 / 43$ & 0.507 \\
\hline Initial AST (IU/l) & $32.83 \pm 14.30$ & $39.91 \pm 15.08$ & 0.001 \\
\hline Follow-up AST (IU/1) & $24.20 \pm 9.27$ & $32.34 \pm 11.04$ & $<0.001$ \\
\hline Initial ALT (IU/l) & $26.13 \pm 19.89$ & $31.39 \pm 17.01$ & 0.058 \\
\hline Follow-up ALT (IU/l) & $26.69 \pm 15.82$ & $33.97 \pm 17.45$ & 0.003 \\
\hline Initial TBIL $(\mu \mathrm{mol} / \mathrm{l})$ & $20.10 \pm 11.55$ & $24.39 \pm 12.63$ & 0.016 \\
\hline Follow-up TBIL $(\mu \mathrm{mol} / \mathrm{l})$ & $18.29 \pm 7.87$ & $24.91 \pm 11.26$ & $<0.001$ \\
\hline Initial PTA (\%) & $89.60 \pm 11.12$ & $76.39 \pm 15.03$ & $<0.001$ \\
\hline Follow-up PTA (\%) & $93.49 \pm 12.60$ & $80.90 \pm 16.42$ & $<0.001$ \\
\hline Initial AFP (ng/ml) & $18.09 \pm 60.50$ & $11.75 \pm 29.64$ & 0.391 \\
\hline Follow-up AFP (ng/ml) & $3.26 \pm 1.94$ & $11.72 \pm 68.30$ & 0.274 \\
\hline Initial HBV DNA (log IU/l) & $2.07 \pm 1.59$ & $2.20 \pm 1.38$ & $<0.001$ \\
\hline Follow-up HBV DNA (log IU/l) & $1.12 \pm 1.51$ & $0.88 \pm 1.19$ & $<0.001$ \\
\hline Initial qHBsAg (IU/ml) & $2,991.54 \pm 5247.24$ & $1,240.17 \pm 1387.61$ & 0.001 \\
\hline Follow-up qHBsAg (IU/ml) & $2,597.24 \pm 5846.97$ & $1,066.22 \pm 1184.79$ & 0.008 \\
\hline Initial LS (kPa) & $14.84 \pm 9.42$ & $21.08 \pm 8.55$ & $<0.001$ \\
\hline Follow-up LS (kPa) & $8.36 \pm 4.88$ & $16.01 \pm 10.60$ & $<0.001$ \\
\hline Therapy before initial LSM (days) & $560.32 \pm 631.21$ & $376.28 \pm 457.63$ & 0.021 \\
\hline Initial platelet count $\left(10^{9} / 1\right)$ & $169.11 \pm 64.19$ & $110.99 \pm 70.15$ & $<0.001$ \\
\hline Initial serum $\mathrm{Cr}(\mu \mathrm{mol} / \mathrm{l})$ & $70.30 \pm 16.66$ & $76.22 \pm 33.05$ & 0.106 \\
\hline Initial BUN (mmol/l) & $4.89 \pm 1.25$ & $4.72 \pm 1.49$ & 0.409 \\
\hline Initial ALB (g/l) & $42.31 \pm 4.48$ & $40.23 \pm 4.53$ & 0.002 \\
\hline Initial triglycerides $(\mathrm{mmol} / \mathrm{l})$ & $1.32 \pm 0.62$ & $1.25 \pm 0.72$ & 0.466 \\
\hline
\end{tabular}

Values are expressed as the mean \pm standard deviation. Normal ranges: AST, 15.0-40.0 IU/l; ALT, 9.0-50.0 IU/1; TBIL, 6.8-30.0 $\mu \mathrm{mol} / \mathrm{l}$; PTA, 80-120\%; AFP, <20 ng/ml; serum Cr, 58-110 $\mu \mathrm{mol} / 1$; BUN, 3.2-7.1 mmol/1; ALB, 40.0-55.0 g/l; triglycerides, 0.28-1.80 mmol/1. qHBsAg, quantitative hepatitis B surface antigen; AST, aspartate aminotransferase; ALT, alanine aminotransferase; TBIL, total bilirubin; AFP, $\alpha$-fetoprotein; PTA, prothrombin time activity; Cr, creatinine; BUN, blood urea nitrogen; ALB, albumin; HBV, hepatitis B virus; LSM, liver stiffness measurement.

In group 1, univariate analysis revealed that factors that may predict a significant reduction of the LS value included lower initial serum TBIL $(\mathrm{P}=0.038)$, higher initial PTA $(\mathrm{P}=0.034)$, higher initial $\mathrm{BUN}(\mathrm{P}=0.026)$ and a higher initial LS $(\mathrm{P}=0.012)$, while multivariate analysis revealed that higher initial LS values $(\mathrm{P}<0.001)$ and a higher PTA $(\mathrm{P}=0.013)$ were correlated with a significant reduction of LS values after adjusting for potential confounders (Table III).

Parameters correlated with the decline of quantitative hepatitis $B$ surface antigen ( $q H B s A g)$. In group 0 , univariate analysis revealed that factors that may predict a significant reduction of the qHBsAg value included female gender $(\mathrm{P}=0.039)$, no family history of HBV infection $(\mathrm{P}=0.029)$ and higher initial qHBsAg $(\mathrm{P}<0.001)$, while multivariate analysis revealed that only the initial qHBsAg value was significantly correlated to the reduction of the qHBsAg values after adjusting for potential confounders $(\mathrm{P}<0.001$; Table IV).

Univariate analysis suggested that factors indicating a greater decline of the LSM value in group 1 included higher initial qHBsAg $(\mathrm{P}<0.001)$ and higher initial triglycerides $(\mathrm{P}=0.020)$. After adjusting for potential confounders, multivariate analysis indicated that higher initial $\mathrm{qHBsAg}$ values were associated with a significant reduction in $\mathrm{qHBsAg}$ values $(\mathrm{P}<0.001$; Table V).

\section{Discussion}

In the present study, the impact of 24 weeks of ETV therapy on LS in 190 CHB patients was investigated. Each patient was evaluated by TE at the beginning and the end of the 24-week period. After ETV treatment, liver injury was found to be significantly improved in each group. In parallel with normalized AST values, HBV DNA titers and LS scores also significantly declined in CHB patients with or without cirrhosis and reduced qHBsAg levels were observed in $\mathrm{CHB}$ patients after 24 weeks of ETV treatment.

LSM is one of the increasingly popular noninvasive methods for detecting and grading liver fibrosis (1). Numerous studies have revealed that this method has good reproducibility, 
Table II. Factors associated with the decline of LS values in uni- and multivariate analyses in group 0.

\begin{tabular}{|c|c|c|c|c|}
\hline Factor & Univariate coefficient & P-value & Multivariate coefficient & P-value \\
\hline Gender & $-1.055^{\mathrm{a}}$ & 0.292 & & \\
\hline Age, years & 0.068 & 0.480 & & \\
\hline Family HBV infection history & $-0.236^{\mathrm{a}}$ & 0.813 & & \\
\hline Initial AST & 0.154 & 0.106 & & \\
\hline Initial ALT & 0.166 & 0.082 & & \\
\hline Initial TBIL & -0.019 & 0.845 & & \\
\hline Initial LS & 0.856 & $<0.001$ & 0.862 & $<0.001$ \\
\hline Initial qHBsAg & -0.130 & 0.173 & & \\
\hline Initial AFP & 0.320 & 0.001 & 0.007 & 0.901 \\
\hline $\mathrm{HBeAg}$ & -0.063 & 0.510 & & \\
\hline Initial HBV DNA (log IU/ml) & 0.126 & 0.187 & & \\
\hline Initial PTA & -0.064 & 0.503 & & \\
\hline Therapy before initial LSM & -0.258 & 0.006 & 0.016 & 0.772 \\
\hline Changes of HBV DNA & 0.268 & 0.005 & 0.057 & 0.300 \\
\hline Initial platelets & -0.094 & 0.327 & & \\
\hline Initial $\mathrm{Cr}$ & 0.060 & 0.535 & & \\
\hline Initial BUN & -0.205 & 0.031 & -0.047 & 0.364 \\
\hline Initial ALB & -0.207 & 0.029 & 0.095 & 0.097 \\
\hline Initial triglycerides & 0.128 & 0.182 & & \\
\hline
\end{tabular}

${ }^{a}$ Mann-Whitney U test. Values are expressed as the mean \pm standard deviation. qHBsAg, quantitative hepatitis B surface antigen; AST, aspartate aminotransferase; ALT, alanine aminotransferase; TBIL, total bilirubin; AFP, $\alpha$-fetoprotein; PTA, prothrombin time activity; Cr, creatinine; BUN, blood urea nitrogen; ALB, albumin; HBV, hepatitis B virus; LSM, liver stiffness measurement; LS, liver stiffness.

Table III. Factors associated with the decline of LS values in uni- and multivariate analyses in group 1.

\begin{tabular}{|c|c|c|c|c|}
\hline Factor & Univariate coefficient & P-value & Multivariate coefficient & $\mathrm{P}$-value \\
\hline Gender & $-1.570^{\mathrm{a}}$ & 0.116 & & \\
\hline Age (years) & -0.180 & 0.112 & & \\
\hline Family HBV infection history & $-0.148^{\mathrm{a}}$ & 0.883 & & \\
\hline Initial AST & 0.021 & 0.885 & & \\
\hline Initial ALT & 0.006 & 0.960 & & \\
\hline Initial TBIL & -0.233 & 0.038 & -0.166 & 0.156 \\
\hline Initial LS & 0.280 & 0.012 & 0.420 & $<0.001$ \\
\hline Initial qHBsAg & -0.011 & 0.926 & & \\
\hline Initial AFP & 0.086 & 0.453 & & \\
\hline $\mathrm{HBeAg}$ & -0.050 & 0.665 & & \\
\hline Initial HBV DNA & 0.032 & 0.779 & & \\
\hline Initial PTA & 0.239 & 0.034 & 0.315 & 0.013 \\
\hline Therapy before initial LSM & 0.100 & 0.380 & & \\
\hline Changes of HBV DNA & 0.045 & 0.695 & & \\
\hline Initial platelets & 0.053 & 0.640 & & \\
\hline Initial $\mathrm{Cr}$ & 0.008 & 0.942 & & \\
\hline Initial BUN & 0.250 & 0.026 & 0.117 & 0.255 \\
\hline Initial ALB & -0.033 & 0.770 & & \\
\hline Initial triglycerides & 0.044 & 0.702 & & \\
\hline
\end{tabular}

${ }^{a}$ Mann-Whitney U test. qHBsAg, quantitative hepatitis B surface antigen; AST, aspartate aminotransferase; ALT, alanine aminotransferase; TBIL, total bilirubin; AFP, $\alpha$-fetoprotein; PTA, prothrombin time activity; Cr, creatinine; BUN, blood urea nitrogen; ALB, albumin; HBV, hepatitis B virus; LSM, liver stiffness measurement, LS, liver stiffness. 
Table IV. Factors associated with the decline of qHBsAg values in uni- and multivariate analyses in group 0.

\begin{tabular}{|c|c|c|c|c|}
\hline Factor & Univariate coefficient & P-value & Multivariate coefficient & P-value \\
\hline Gender & -2.064 & 0.039 & -0.099 & 0.257 \\
\hline Age (years) & 0.016 & 0.889 & & \\
\hline Family HBV infection history & $-2.180^{\mathrm{a}}$ & 0.029 & -0.105 & 0.227 \\
\hline Initial AST & -0.166 & 0.082 & & \\
\hline Initial ALT & -0.092 & 0.337 & & \\
\hline Initial Total bilirubin & -0.053 & 0.579 & & \\
\hline Initial LS & -0.074 & 0.441 & & \\
\hline Initial qHBsAg & 0.462 & $<0.001$ & 0.460 & $<0.001$ \\
\hline Initial AFP & -0.045 & 0.639 & & \\
\hline $\mathrm{HBeAg}$ & 0.044 & 0.645 & & \\
\hline Initial HBV DNA & 0.158 & 0.099 & & \\
\hline Initial PTA & 0.049 & 0.611 & & \\
\hline Therapy before initial LSM & 0.017 & 0.857 & & \\
\hline Changes of HBV DNA & 0.011 & 0.910 & & \\
\hline Initial platelets & 0.008 & 0.932 & & \\
\hline Initial $\mathrm{Cr}$ & 0.062 & 0.519 & & \\
\hline Initial BUN & 0.097 & 0.310 & & \\
\hline Initial ALB & 0.100 & 0.294 & & \\
\hline Initial triglycerides & 0.005 & 0.962 & & \\
\hline
\end{tabular}

${ }^{a}$ Mann-Whitney U test. qHBsAg, quantitative hepatitis B surface antigen; AST, aspartate aminotransferase; ALT, alanine aminotransferase; TBIL, total bilirubin; AFP, $\alpha$-fetoprotein; PTA, prothrombin time activity; Cr, creatinine; BUN, blood urea nitrogen; ALB, albumin; HBV, hepatitis B virus; LSM, liver stiffness measurement.

Table V. Factors associated with the decline of qHBsAg in uni- and multivariate analyses in group 1.

\begin{tabular}{|c|c|c|c|c|}
\hline Factor & Univariate coefficient & P-value & Multivariate coefficient & P-value \\
\hline Gender & $-1.377^{\mathrm{a}}$ & 0.169 & & \\
\hline Age, years & 0.002 & 0.985 & & \\
\hline Family HBV infection history & $-0.787^{\mathrm{a}}$ & 0.431 & & \\
\hline Initial AST & 0.035 & 0.762 & & \\
\hline Initial ALT & -0.064 & 0.573 & & \\
\hline Initial Total bilirubin & -0.057 & 0.619 & & \\
\hline Initial LS & -0.213 & 0.059 & & \\
\hline Initial qHBsAg & 0.526 & $<0.001$ & 0.420 & $<0.001$ \\
\hline Initial AFP & -0.018 & 0.878 & & \\
\hline $\mathrm{HBeAg}$ & -0.076 & 0.506 & & \\
\hline Initial HBV DNA (IU/ml) & 0.019 & 0.871 & & \\
\hline Initial PTA & 0.083 & 0.467 & & \\
\hline Therapy before initial LSM & -0.034 & 0.767 & & \\
\hline Changes of HBV DNA & 0.069 & 0.543 & & \\
\hline Initial platelets & 0.086 & 0.453 & & \\
\hline Initial $\mathrm{Cr}$ & -0.108 & 0.344 & & \\
\hline Initial BUN & -0.109 & 0.337 & & \\
\hline Initial ALB & -0.075 & 0.513 & & \\
\hline Initial triglycerides & 0.261 & 0.020 & 0.138 & 0.165 \\
\hline
\end{tabular}


relative reflection of histology and clinical utility $(4,6,8,9)$. However, the stiffness in hepatic parenchyma may be caused by necroinflammation and/or fibrosis. LSM is not able to distinguish necroinflammation from fibrosis (1). In addition, the changes in LS values in severe inflammation at baseline did not reflect real reversal of fibrosis $(8,10,11)$. Accordingly, patients with AST $>100 \mathrm{IU} / 1$ or ALT $>100 \mathrm{IU} / 1$ at baseline were excluded for minimizing the influence of inflammatory activity on LS values. Furthermore, it was identified that in parallel with normalized AST values, LS scores significantly declined in CHB patients with or without cirrhosis after 24 weeks of ETV treatment.

The present findings regarding the LS scores were principally consistent with previously published data. For instance, liver fibrosis has been reported to improve in $35-38 \%$ of lamivudine-treated CHB patients and 36-39\% of ETV-treated CHB patients after one year $(12,13)$. Long-term ETV treatment ( $>3$ years) attenuated necroinflammatory activity in all patients and reduced liver fibrosis in $57-100 \%$ of patients $(1,14)$. Furthermore, advanced liver fibrosis was shown to be reversed after long-term antiviral treatment $(14,15)$. However, these previous studies always included patients with long-term antiviral treatment of at least $>1$ year. However, in clinical practice, patients with advanced fibrosis may not receive long-term antiviral treatment due to the occurrence of CHB-associated complications and the uncertainty of the survival time. However, according to our experience, short-term antiviral treatment may not show an obvious benefit. Based on the results of the present study, it may be concluded that hepatic fibrosis may regress in patients with liver cirrhosis after only 24 weeks of ETV treatment. As liver fibrosis can be reduced in a short time, CHB patients in different stages may all benefit from antiviral treatment. However, the present study had various limitations; for instance, the patients with a Child Pugh Score of $\geq 9$ or ascites were not included as LS values may be influenced by ascites.

The present study found that the substantially reduced LS values in the two groups after 24 weeks of ETV treatment were associated with higher baseline LS scores, as has been previously reported $(10,16)$. The reason for this may be that patients with higher initial LS values had a greater chance of reduction in LS values. In addition, it may be hypothesized that a significant decline in LS values may reflect an improvement in liver fibrosis after ETV treatment, as the influence of high ALT levels was excluded. Beyond that, patients with a shorter duration of antiviral treatment showed a larger decline of their LS value at follow-up, which may have been due to their comparatively worse liver condition when they were recruited. This noteworthy correlation was in agreement with the findings by Kuo et al (10). Therefore, the present study showed that patients with higher liver fibrosis levels should receive antiviral therapy, as it achieves a greater improvement in $\mathrm{LS}$ values.

Certain studies reevaluated the pathogenesis of HBV infection and suggested that a high level of HBV replication in infected cells leads to a high level of intracellular accumulation of viral products, which causes the destruction of infected cells. Inhibition of replication- and accumulation-mediated liver injury provides an explanation for the efficacy of nucleos $(t) i d e$ analogue therapy in mitigating liver injury and reducing liver fibrosis in CHB patients. According to this mechanism, it is inferred that the decline of HBV DNA titers is associated with the reduction of LS values. However, the results of the present study did not identify any independent correlation between the changes of HBV DNA with the significant reduction in LSM values after adjusting for potential confounders in CHB patients receiving ETV treatment. This may be due to exclusion of patients with severe inflammation at baseline, which diminished the significance of a decline of liver fibrosis and inflammation induced by reduction of HBV DNA titers. In addition, the interval between the two LSMs in the present study was 24 weeks, while the effectiveness in reducing liver fibrosis caused by suppression of viral replication may be exerted over a longer period, which requires assessment in future studies. HBsAg was initially discovered and named 'Australia antigen' in 1968 (17) and is the hallmark of HBV infection. HBsAg levels have been reported to be in parallel with intra-hepatic HBV DNA and covalently closed circular DNA (cccDNA) levels. In the present study, the patient in the two groups did not achieve a significant decline of qHBsAg during 24 weeks of treatment with ETV. This may be due to a lack of a direct effect of nucleos(t)ide analogues on cccDNA and the fact that the intensity of host immunity to reduce the qHBsAg levels was low in the patients with ALT $\leq 2$ times the upper limit of normal. Furthermore, the mean duration of antiviral treatment before initial LS was 484 days in the present study, which is when the effect of the immune reaction decreases (18-20). Previous studies reported that the baseline qHBsAg predicts the treatment or virologic response in CHB patients with nucleos $(t)$ ide treatment $(18,19)$. In the present study, the substantially reduced qHBsAg values in the two groups after 24 weeks of ETV treatment were correlated with a higher initial qHBsAg but not with LS values. This suggested that the decline of qHBsAg was associated with HBV itself rather than the severity of patients' illness when the intensity of host immunity was low and patients with higher initial qHBsAg benefited more from antiviral treatment.

In conclusion, the present study evaluated changes in LS in 190 CHB patients who received ETV treatment and found a significant decrease in LSM scores in patients with or without cirrhosis only after 24 weeks of ETV treatment. The results suggested that higher LS values at baseline contributed to a greater LS regression and higher initial qHBsAg values led to a greater reduction of qHBsAg during ETV therapy. Based on these results, ETV treatment provided a greater benefit for CHB patients with higher initial LS values or qHBsAg levels at baseline.

\section{Acknowledgements}

The authors would like to thank Dr Hong Zhang for his assistance with the statistical evaluation of the data.

\section{References}

1. Osakabe K, Ichino N, Nishikawa T, Sugiyama H, Kato M, Kitahara S, Hashimoto S, Kawabe N, Harata M, Nitta Y, et al: Reduction of liver stiffness by antiviral therapy in chronic hepatitis B. J Gastroenterol 46: 1324-1334, 2011.

2. Su TH, Kao JH and Liu CJ: Molecular mechanism and treatment of viral hepatitis-related liver fibrosis. Int J Mol Sci 15: 10578-10604, 2014. 
3. Lee S and Kim DY: Non-invasive diagnosis of hepatitis B virus-related cirrhosis. World J Gastroenterol 20: 445-459, 2014.

4. Friedrich-Rust M, Ong MF, Martens S, Sarrazin C, Bojunga J, Zeuzem S and Herrmann E: Performance of transient elastography for the staging of liver fibrosis: A meta-analysis. Gastroenterology 134: 960-974, 2008.

5. Barreiro P, Labarga P, Martín-Carbonero L, Amor A, Ruiz-Sancho A, Castellares C, González-Lahoz J and Soriano V: Sustained virological response following HCV therapy is associated with non-progression of liver fibrosis in $\mathrm{HCV} /$ HIV-coinfected patients. Antivir Ther 11: 869-877, 2006.

6. Sandrin L, Fourquet B, Hasquenoph JM, Yon S, Fournier C, Mal F, Christidis C, Ziol M, Poulet B, Kazemi F, et al: Transient elastography: A new noninvasive method for assessment of hepatic fibrosis. Ultrasound Med Biol 29: 1705-1713, 2003.

7. Jung KS and Kim SU: Clinical applications of transient elastography. Clin Mol Hepatol 18: 163-173, 2012.

8. Coco B, Oliveri F, Maina AM, Ciccorossi P, Sacco R, Colombatto P, Bonino F and Brunetto MR: Transient elastography: A new surrogate marker of liver fibrosis influenced by major changes of transaminases. J Viral Hepat 14: 360-369, 2007.

9. Kim SU, Park JY, Kim DY, Ahn SH, Choi EH, Seok JY, Lee JM, Park YN, Chon CY and Han KH: Non-invasive assessment of changes in liver fibrosis via liver stiffness measurement in patients with chronic hepatitis B: Impact of antiviral treatment on fibrosis regression. Hepatol Int 4: 673-680, 2010.

10. Kuo YH, Lu SN, Chen CH, Chang KC, Hung CH, Tai WC Tsai MC, Tseng PL, Hu TH and Wang JH: The changes of liver stiffness and its associated factors for chronic hepatitis $\beta$ patients with entecavir therapy. PLoS One 9: e93160, 2014

11. Oliveri F, Coco B, Ciccorossi P, Colombatto P, Romagnoli V, Cherubini B, Bonino F and Brunetto MR: Liver stiffness in the hepatitis B virus carrier: A non-invasive marker of liver disease influenced by the pattern of transaminases. World $\mathrm{J}$ Gastroenterol 14: 6154-6162, 2008.
12. Chang TT, Gish RG, de Man R, Gadano A, Sollano J, Chao YC, Lok AS, Han KH, Goodman Z, Zhu J, et al: A comparison of entecavir and lamivudine for HBeAg-positive chronic hepatitis B. N Engl J Med 354: 1001-1010, 2006.

13. Lai CL, Shouval D, Lok AS, Chang TT, Cheinquer H, Goodman Z, DeHertogh D, Wilber R, Zink RC, Cross A, et al: Entecavir versus lamivudine for patients with $\mathrm{HBeAg}$-negative chronic hepatitis B. N Engl J Med 354: 1011-1020, 2006.

14. Schiff ER, Lee SS, Chao YC, Kew Yoon S, Bessone F, Wu SS Kryczka W, Lurie Y, Gadano A, Kitis G, et al: Long-term treatment with entecavir induces reversal of advanced fibrosis or cirrhosis in patients with chronic hepatitis B. Clin Gastroenterol Hepatol 9: 274-276, 2011

15. Calvaruso V and Craxì A: Regression of fibrosis after HBV antiviral therapy. Is cirrhosis reversible? Liver Int 34 (Suppl 1): S85-S90, 2014.

16. Kim BK, Fung J, Yuen MF and Kim SU: Clinical application of liver stiffness measurement using transient elastography in chronic liver disease from longitudinal perspectives. World J Gastroenterol 19: 1890-1900, 2013.

17. Blumberg BS, Sutnick AI and London WT: Hepatitis and leukemia: Their relation to Australia antigen. Bull NY Acad Med 44: 1566-1586, 1968.

18. Wang CC, Tseng TC, Wang PC, Lin HH and Kao JH: Baseline hepatitis $B$ surface antigen quantitation can predict virologic response in entecavir-treated chronic hepatitis B patients. J Formos Med Assoc 113: 786-793, 2014.

19. Chen $\mathrm{CH}$, Chiu YC, Lu SN, Lee CM, Wang JH, Hu TH and Hung $\mathrm{CH}$ : Serum hepatitis B surface antigen levels predict treatment response to nucleos(t)ide analogues. World J Gastroenterol 20: 7686-7695, 2014.

20. Tseng TC and Kao JH: Clinical utility of quantitative HBsAg in natural history and nucleos(t)ide analogue treatment of chronic hepatitis B: New trick of old dog. J Gastroenterol 48: 13-21, 2013. 